\title{
Theoretical study of the electronic, structural, and cohesive properties of ruthenium
}

\author{
James R. Chelikowsky \\ Corporate Research Science Laboratories, Exxon Research and Engineering Company, Annandale, New Jersey 08801 \\ C. T. Chan \\ Department of Physics, Ames Laboratory, Iowa State University, Ames, Iowa 50010
}

Steven G. Louie

Department of Physics, University of California, Berkeley, California 94720

and Materials and Molecular Research Division, Lawrence Berkeley Laboratory, University of California, Berkeley, California 94720

(Received 29 May 1986)

\begin{abstract}
The electronic, structural, and cohesive properties of ruthenium in the hexagonal-close-packed structure are calculated by using a local-orbital $a b$ initio pseudopotential method. We determine the lattice parameters, Poisson's ratio, the cohesive energy, and the bulk modulus by calculating the total energy of the solid-state system as a function of the lattice parameters $c$ and $a$. We also predict the value of the $c / a$ ratio up to pressures of $1 \mathrm{Mbar}$. We find that as a function of pressure the $c / a$ value approaches the ideal value. Overall, the agreement with the observed lattice parameters is quite good. The largest structural error is in the $c$ lattice parameter and is approximately $2 \%$. The cohesive energy is larger than experiment by approximately $15 \%$ and is consistent with other localdensity calculations for transition metals. The calculated band structure is in accord with photoemission measurements.
\end{abstract}

\section{INTRODUCTION}

A number of methods has been developed to calculate, from first principles, the total energy of solid-state systems. $^{1-15}$ A common element of many of these methods is to incorporate the use of ab initio pseudopotentials. ${ }^{5,7,12-15}$ Ab initio pseudopotential methods using plane-wave bases have been applied to a number of simple metal, semiconductor, and insulating systems with good success, e.g., these methods have accurately predicted phase transitions, structural properties, bulk moduli, phonon dispersions, and elastic constants. ${ }^{12-16}$ Extending these methods to incorporate Gaussian bases has made them applicable to a variety of solid-state systems including not only the semiconductor systems, but also transition-metal systems. ${ }^{15,17}$

In this paper we will use a pseudopotential description of the electronic properties and a Gaussian basis to examine the structural, electronic, and cohesive properties of ruthenium metal. We have chosen to examine ruthenium metal for several reasons. First, the band structure of the material is fairly well known from a variety of different calculations. ${ }^{1,18-21}$ Thus, we can readily compare our method of calculation with other methods. Second, ruthenium metal is an efficient catalyst for methanation and it is often used in combination with other metals as a commercial catalyst. ${ }^{22-25}$ It would be useful to establish a theoretical framework for its bulk structural properties in order to examine more closely its surface properties and compare to other transition metals. Third, ruthenium in its ground state has a noncubic structure, i.e., hexagonal close packed. The hexagonal-close-packed structure is the most common among the transition metals, yet most calculations on transition metals have concentrated on cubic bulk materials. These calculations have been very successful and one would expect similar results would be obtained for a noncubic material.

\section{METHODS OF CALCULATION}

Our calculations are based on determining the total energy of the solid-state system through a solution of a selfconsistent-field single-particle Schrödinger equation within a local-density approximation for exchange and correlation. The details of our approach have been discussed elsewhere ${ }^{14,15,17}$ here we briefly review our method and give computational details.

The first step in the calculation is to construct an $a b$ initio ionic pseudopotential for ruthenium. Our construction follows the work of Hamann et al. ${ }^{26}$ The only input into this calculation involves the atomic number and a core length scale which delineates the transition from "corelike" to "valencelike" regions of the atomic species. Outside of the core we demand that the pseudopotential and the all-electron potential yield identical wave functions for the atom. Inside the core, we demand the pseudo wave functions are nodeless and contain the same amount of charge as the all-electron wave functions. The real potential and pseudopotential atomic eigenvalues are fixed to be identical. The atomic configuration for the pseudopotential construction was taken to be $5 s^{1} 4 d^{7}$. The core length scales for $s, p$, and $d$ components were taken to be $1.7,1.7,0.7$ in atomic units.

The ionic pseudopotential determined as indicated above was expressed as follows: 


$$
V_{\text {ion }}(\mathbf{r})=V_{L}(\mathbf{r})+\sum_{l=0}^{1}\left[V_{l}(\mathbf{r})-V_{L}(\mathbf{r})\right]|l\rangle\langle l|,
$$

where angular momentum components up to $l=2$ were considered, i.e., $V_{s}-V_{L}$ and $V_{p}-V_{L}$ are used as nonlocal "corrections" to the local potential $V_{L}=V_{d}$. To initiate the calculation, atomic pseudocharge densities were superimposed to form an approximate crystalline charge density $\rho_{\text {crystal }}^{a}$, from which a Hartree potential $V_{H}$ and an exchange-correlation local-density approximation potential $V_{\mathrm{xc}}$ can be constructed. These two potentials are decomposed into a linear combination of on-site effective screening potentials which are summed with the ionic local potential to form a neutral local potential:

$$
V_{\mathrm{loc}}(r)=V_{L}(r)+V_{H}^{\mathrm{eff}}\left(\rho_{\text {crystal }}^{a}, r\right)+V_{\mathrm{xc}}^{\mathrm{eff}}\left(\rho_{\text {crystal }}^{a}, r\right) .
$$

The total crystalline potential consists of a sum over these atomic centered potentials plus the nonlocal terms. For many materials, the superposition of atomic charge densities is not a bad approximation to the self-consistent charge in a crystal and $V_{\text {loc }}$ is a reasonable starting potential.

In order to expedite the evaluation of matrix elements required for a solution of the single-particle Schrödinger equation, we express both $V_{\text {loc }}$ and $V_{l}-V_{L}$ in terms of Gaussians of the form

$$
V(r)=\sum_{i} c_{i} \exp \left(-\beta_{i} r^{2}\right)
$$

This expansion allows us to express all two- and threecenter integrals in terms of analytic summations. The determination of the coefficients and Gaussian decay constants is nontrivial as it involves a nonlinear, multiparameter optimization process. We used a Monte Carlo simulated annealing technique, ${ }^{27}$ the fitted values for $c_{i}$ and $\beta_{i}$ are given in Table I. We note that these initial potentials yield a reasonable band structure, e.g., within $0.5 \mathrm{eV}$ of the self-consistent band structure.

Our basis consisted of a Bloch sum of Gaussian orbitals which has the form

$$
\Phi_{i}(\mathbf{k}, \mathbf{r})=\frac{1}{\sqrt{\Omega}} \sum_{\mathbf{R}} \exp \left[i \mathbf{k}\left(\mathbf{R}+\tau_{\mu}\right)\right] f_{\alpha l m}\left(\mathbf{r}+\mathbf{R}+\tau_{\mu}\right),
$$

where $i=\{\operatorname{lm} \mu \alpha\}$ is a composite index, $\Omega$ is the crystal volume, $\mathbf{R}$ is a lattice vector, $\tau_{\mu}$ is a basis vector, and $f(\mathbf{r})$ are Gaussian-type functions of the form

$$
f_{\alpha l m}(\mathbf{r})=A_{\alpha l m} \exp \left(-\alpha r^{2}\right) r^{l} K_{l m}(\theta, \phi),
$$

where $A_{\alpha l m}$ 's are normalization constants. "Kubic harmonics" $\left[K_{l m}(\theta, \phi)\right]$ up to $l=2(s, p, d)$ are included. To determine the decay constants $(\alpha)$, we minimized the total energy of the system. We found that four constants were necessary to achieve reasonable convergence, i.e., the total energy converged to within $0.1 \mathrm{eV}$ in absolute magnitude. The decays we used are $\alpha=0.25,0.5723,1.3104$, and 3.0. In optimizing these decays, we altered the shortest and longest decays and constrained the middle two to be even tempered. ${ }^{14}$ These decays are somewhat similar to those used for W and Mo. ${ }^{17}$ The Hamiltonian matrix sizes are $80 \times 80$ for the hexagonal-close-packed structure as we

\begin{tabular}{|c|c|}
\hline$c_{i}$ & $\beta_{i}$ \\
\hline \multicolumn{2}{|c|}{$V_{\mathrm{loc}}$} \\
\hline-1.65295 & 0.20606 \\
\hline-0.71121 & 0.56186 \\
\hline-14.68993 & 0.84649 \\
\hline 89.44192 & 2.00001 \\
\hline-302.71853 & 2.63232 \\
\hline 377.77770 & 3.70162 \\
\hline-95.40590 & 4.96774 \\
\hline-118.84195 & 5.28692 \\
\hline 57.93663 & 6.97335 \\
\hline 1.07606 & 47.70755 \\
\hline \multicolumn{2}{|c|}{$V_{p}-V_{L}$} \\
\hline 38.86179 & 0.84391 \\
\hline-44.92577 & 1.28821 \\
\hline 171.07063 & 2.64773 \\
\hline-115.60520 & 3.60955 \\
\hline-156.70623 & 4.15618 \\
\hline 154.30146 & 5.62370 \\
\hline-210.99365 & 11.95607 \\
\hline 226.11115 & 13.43778 \\
\hline-49.62542 & 16.78450 \\
\hline-0.28287 & 69.54027 \\
\hline \multicolumn{2}{|c|}{$V_{s}-V_{L}$} \\
\hline-23.04885 & 0.62329 \\
\hline 64.00733 & 0.77237 \\
\hline-93.10525 & 1.50066 \\
\hline 49.38465 & 1.88528 \\
\hline 130.27535 & 2.40737 \\
\hline-219.24891 & 4.00388 \\
\hline 167.38859 & 5.84821 \\
\hline-80.71093 & 8.69784 \\
\hline 22.97639 & 11.58617 \\
\hline-1.96357 & 35.61035 \\
\hline
\end{tabular}

TABLE I. Coefficients $\left(c_{i}\right)$ in rydbergs and exponential decays $\left(\beta_{i}\right)$ in a.u. ${ }^{-2}$ for the Gaussian expansion of the local and nonlocal components of the ruthenium potential [see Eq. (3) and text].

have two atoms per unit cell and 40 Gaussian-like orbitals per atom.

To achieve self-consistency, we use a momentum space scheme. ${ }^{17}$ The first step in this process is to evaluate the overlap matrix, $\Pi_{i j}(\mathbf{k}, \mathbf{G})$ :

$$
\mathrm{II}_{i j}(\mathbf{k}, \mathbf{G})=\left\langle\mathbf{k}, j\left|\exp (-i \mathbf{G} \cdot \mathbf{r}) / \Omega_{c}\right| \mathbf{k}, i\right\rangle,
$$

where $\Omega_{c}$ is the volume of the unit cell, $\mathbf{G}$ are reciprocallattice vectors, and $|\mathbf{k}, i\rangle$ are the basis orbitals given in Eq. (4). These overlap matrices are used to compute the charge density and the changes of the Hamiltonian matrix elements, $\delta H_{i j}(\mathbf{k})$, at a $\mathbf{k}$ point for an arbitrary change in the crystal potential $\delta V$, e.g., $\delta V=V_{\text {scr }}($ out $)-V_{\text {scr }}$ (in) where $V_{\text {scr }}$ is the sum of the Hartree and exchange correlation potentials. More specifically, we can write

$$
\rho(G)=\sum_{n, \mathbf{k}} \psi_{n \mathbf{k}}^{\dagger} \Pi(\mathbf{k}, \mathbf{G}) \psi_{n \mathbf{k}}
$$

and 


$$
\delta H_{i j}(\mathbf{k})=\sum_{\mathbf{G}} \delta V(G) \Pi_{i j}^{*}(\mathbf{k}, \mathbf{G}) \Omega_{c},
$$

where we use matrix notation in (7); $\psi_{n \mathbf{k}}$ is the column vector in the basis space corresponding to a wave function. In this formalism, there is no need to fit the potential to Gaussians during the self-consistency iteration process and the final self-consistent potential has no special shape constraint within the chosen basis.

As for other computational details, the local-density approximation for exchange and correlation is taken from Hedin and Lundqvist. ${ }^{28}$ The number of $\mathbf{k}$ points used to determine the charge density and the Fermi level was taken to be 18 points over a uniform grid in the irreducible Brillouin zone. We have also tested a grid of 40 points and determined that the total energy varied by less than $0.02 \mathrm{eV}$, and with respect to changes in structural energies the errors are estimated to be an order of magnitude smaller. Gaussian weighting in the occupancy of the electronic states near the Fermi level was used to increase the stability of the total energy with respect to the number of k points sampled. ${ }^{29}$ Iterations were carried out until the Fourier components of the potentials were self-consistent to at least $0.1 \mathrm{mRy}$. For a given set of $\mathbf{k}$ points, the total energy is stable to within $10^{-5} \mathrm{eV}$. As far as computing the matrix elements in real space, i.e., overlap and threecenter integrals, typically we included up to the tenthnearest-neighbor shell.

To evaluate the total energy of the system from a knowledge of the wave functions and the eigenvalues, we use the momentum space formalism of Ihm et al. ${ }^{11}$ In this method, the Gaussian wave functions are expanded into plane waves and the relevant reciprocal space summations formed. We included all reciprocal-lattice vectors whose magnitude is less than 8.0 a.u. For the known lattice parameters of ruthenium, this criterion produces sums which include 1497 plane-wave terms.

\section{STRUCTURAL AND COHESIVE PROPERTIES}

The structural properties of hexagonal-close-packed ruthenium are more complicate than cubic materials as we must optimize both the $c$ and $a$ parameters to establish the lowest-energy configuration. To perform this task we constructed a grid of points in $c$ and $a$ space and evaluated the total energy over this grid. We then fit a simple quadratic expansion of the total energy in this space to determine $c$ and $a$. Similar procedures have been followed for other hexagonal-close-packed metals. ${ }^{16}$ The form of our expansion is given by

$$
\begin{aligned}
E(c, a)= & E_{0}+\alpha\left(a-a_{0}\right)^{2}+\beta\left(c-c_{0}\right)^{2} \\
& +\gamma\left(a-a_{0}\right)\left(c-c_{0}\right),
\end{aligned}
$$

where $E_{0}$ is the equilibrium energy; $c_{0}$ and $a_{0}$ are the equilibrium lattice parameters; and $\alpha, \beta$, and $\gamma$ are parameters which control the relative compressibility along the $c$ and $a$ axes. We evaluated the energy $E(c, a)$ over a grid of nine different values of $c$ and $a$ to determine the six parameters in (9). As a reference energy we considered the energy of the isolated pseudoatom. The difference be- tween this energy and our solid-state system will yield the cohesive energy. The energy of the atom was calculated to be $-447.16 \mathrm{eV}$. This value included a spin polarization correction ${ }^{30}$ of $1.80 \mathrm{eV}$. Since ruthenium is not magnetic, we did not consider spin polarization corrections to the solid. In Table II we present the grid in $c$ and $a$ space, the calculated values of $E(c, a)$ and the resulting values for $E_{0}, a_{0}, c_{0}, \alpha, \beta$, and $\gamma$. Our fit over the given range of $c$ and $a$ lattice parameters was better than 0.01 eV.

While the bulk modulus for ruthenium can be extracted from (9), we do not expect this value to be especially reliable, since we have assumed no terms in (9) which correspond to anharmonic deviations of $E(c, a)$ and the grid spacings in $c$ and $a$ space which we used are fairly large. In order to evaluate the bulk modulus, we examined $E(\Omega)$ as a function of volume, $\Omega$, where for a given volume we took the lowest-energy values of $c$ and $a$ as estimated from (9). In Table III we list $E(\Omega)$ as a function of volume. We fit this curve to a Murnaghan equation of state, ${ }^{31}$ and in Fig. 1 we illustrate the accuracy of our fit.

In Table IV we present the calculated structural and cohesive properties of ruthenium. Overall, the agreement for the calculated and observed lattice constants are quite good. The error for the $c$ parameter of approximately $2 \%$ is a bit larger than for cubic other materials, e.g., Mo or $\mathrm{W}$; however, this error is not so large as to indicate an inherent problem with our approach. The calculated cohesive energy is not expected to be very accurate as we must subtract the difference between two rather large numbers to determine the binding energy. Despite this problem, the error we make in the cohesive energy of ruthenium is about $15 \%$. Moreover, the error is on the side of yielding a cohesive energy which is too large. This

TABLE II. $E(c, a)$ in $\mathrm{eV}$ referenced to the free ruthenium pseudoatom. The fitting parameters are taken from Eq. (9). The units are such that if $c$ and $a$ are given in units of the ex-

\begin{tabular}{|c|c|c|c|c|}
\hline$c / c_{\text {expt }}$ & & $a / a_{\text {expt }}$ & & $E(c, a)$ \\
\hline 1.00 & & 1.05 & & -7.5226 \\
\hline 1.00 & & 1.00 & & -7.6956 \\
\hline 1.00 & & 0.95 & & -7.6535 \\
\hline 0.95 & & 1.05 & & -7.5459 \\
\hline 0.95 & & 1.00 & & -7.7279 \\
\hline 0.95 & & 0.95 & & -7.6105 \\
\hline 0.90 & & 1.05 & & -7.5225 \\
\hline 0.90 & & 1.00 & & -7.5734 \\
\hline \multirow[t]{8}{*}{0.90} & & 0.95 & & -7.3561 \\
\hline & $\begin{array}{c}\text { Fitting } \\
\text { parameters }\end{array}$ & & $\begin{array}{l}\text { Resulting } \\
\text { values }\end{array}$ & \\
\hline & $E_{0}$ & & -7.7376 & \\
\hline & $\alpha$ & & 56.86 & \\
\hline & $\beta$ & & 34.33 & \\
\hline & $\gamma$ & & 36.76 & \\
\hline & $c_{0}$ & & 0.972 & \\
\hline & $a_{0}$ & & 0.990 & \\
\hline
\end{tabular}
perimental values $\left(c_{\text {expt }}=4.28 \AA\right.$ and $\left.a_{\text {expt }}=2.71 \AA\right)$, then $E(c, a)$ is in $\mathrm{eV}$. 
TABLE III. $E\left(\Omega_{a}\right)$ as a function of atomic volume $\left(\Omega_{a}\right)$ in a.u. The minimum energy value of $c$ and $a$ as determined from Eq. (9) are also given.

\begin{tabular}{rclc}
\hline \hline$\Omega_{a}$ & $c / c_{\text {expt }}$ & $a / a_{\text {expt }}$ & $E\left(\Omega_{a}\right)$ \\
\hline 72.5 & 0.945 & 0.9139 & -7.2187 \\
77.5 & 0.953 & 0.941 & -7.5530 \\
82.5 & 0.964 & 0.967 & -7.7150 \\
90.0 & 0.977 & 1.001 & -7.7321 \\
100.0 & 0.998 & 1.045 & -7.4946 \\
\hline \hline
\end{tabular}

is consistent with the trend observed for a number of local-density calculations for transition metals involving different bases and all electron potentials. ${ }^{32}$ Thus, the error appears to be an inherent problem associated with the local-density approach.

The calculated bulk modulus is in good accord with experiment. We note that for the early transition metals, it has been shown that one needs to incorporate explicit valence-core contributions to exchange correlation in the pseudopotential approach. ${ }^{33}$ This effect arises from a fairly sizable core-valence wave-function overlap. Here we do not include these effects and yet our calculation yields a satisfactory bulk modulus. This is consistent with previous work suggesting that this correction to the bulk modulus should only be large for the early transition metals.

For Poisson's ratio, ${ }^{34}$ i.e., the negative ratio of the transverse strain to the corresponding axial strain in a body subject to uniaxial stress, we need to compute the value of $a$ for a given value of $c$. The information contained in Table II is sufficient for this purpose. For three values of $c\left(0.9,0.95\right.$, and 1.0 in units of $\left.c_{\text {expt }}\right)$, we fit a parabola through the energy calculated at three $a$ points $\left(0.95,1.0\right.$, and 1.05 in units of $\left.a_{\text {expt }}\right)$. The results are shown in Fig. 2, where we have fit a linear function for $a$ versus $c$. Expressing Poisson's ratio $\sigma$ as

$$
\sigma=-(\Delta a / a) /(\Delta c / c)
$$

the value extracted from Fig. 2 is 0.315 . However, we can

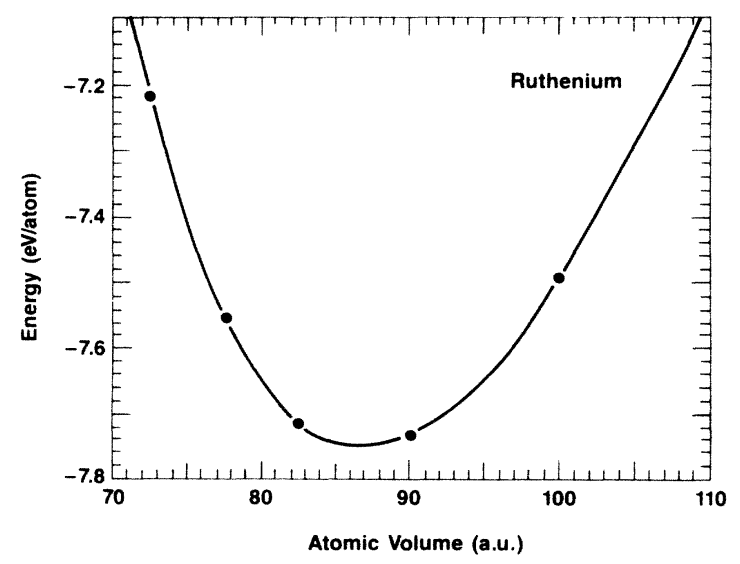

FIG. 1. Calculated total energy of hexagonal-close-packed ruthenium as a function of atomic volume. The solid line represents a Murnaghan equation of state fitted to the calculated points.
TABLE IV. Comparison of the ground-state properties of ruthenium in the hexagonal-close-packed structure. Experimental data are from C. Kittel, Introduction to Solid State Physics (Wiley, New York, 1976); K. Geschneidner, Jr., in Solid State Physics, edited by H. Ehrenreich, F. Seitz, and D. Turnbull (Academic, New York, 1964), Vol. 16, p. 275; and R. W. G. Wyckoff, Crystal Structures, 2nd ed. (Interscience, New York, 1963). The cohesive energy has been corrected for zero-point motion as suggested in Ref. 1.

\begin{tabular}{ccc}
\hline \hline \multicolumn{1}{c}{ Property } & Experiment & Theory \\
\hline Cohesive energy $(\mathrm{eV})$ & 6.62 & 7.70 \\
$a(\AA)$ & 2.71 & 2.68 \\
$c(\AA)$ & 4.28 & 4.16 \\
$c / a$ & 1.579 & 1.552 \\
Bulk modulus (Mbar) & 3.21 & 3.51 \\
Poisson ratio & 0.29 & 0.31 \\
$(\partial B / \partial P)_{P=0}$ & & 4.42 \\
\hline \hline
\end{tabular}

also extract a value using Eq. (9) and the data in Tables II and III. This value tends to be a bit lower, i.e., 0.308. Within the errors of calculation, we feel the agreement between the estimated value of 0.29 from Gschneidner (Table IV) and our calculated values is satisfactory.

Another measure of the structural parameters is to extract the minimum energy value of $c / a$ as a function of pressure. Here we do not have experimental data. Nonetheless, we expect our prediction in Fig. 3 is accurate given our relatively small errors for the other structural properties. The calculated value is plotted as a function of pressure given by

$$
P(\Omega)=\left(B_{0} / B_{0}^{\prime}\right)\left[\left(\Omega_{0} / \Omega\right)^{B_{0}^{\prime}}-1\right],
$$

where the values of $B_{0}$ and $B_{0}^{\prime}$, the bulk modulus and pressure derivative of the bulk modulus, are taken from Table III. We have plotted the value of $c / a$ versus this pressure with the minimized $c / a$ value for a given pressure (or volume) obtained from $E(c, a)$ as given by Eq. (9). We do not expect $E(c, a)$ as expressed by (9) to be valid for very large pressures, or small volumes, as it does not

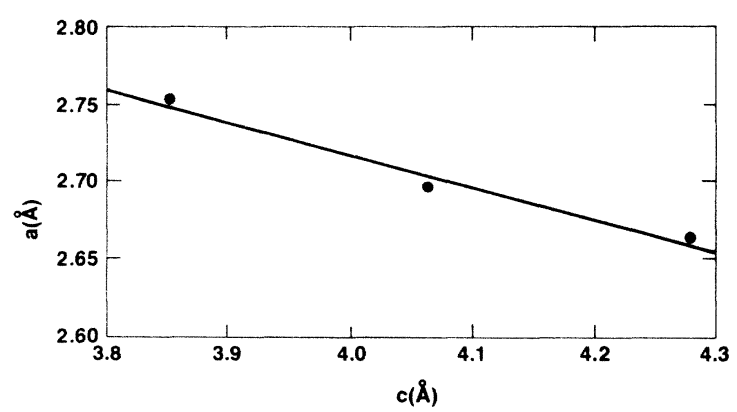

FIG. 2. The lattice parameter $a$ as a function of $c$. The lowest-energy value of $a$ for a given value of $c$ has been determined from the data in Table II for three values of $c$. A linear relationship between $c$ and $a$ is shown in the figure. From a knowledge of $a$ vs $c$, it is possible to determine Poisson's ratio (see text). 


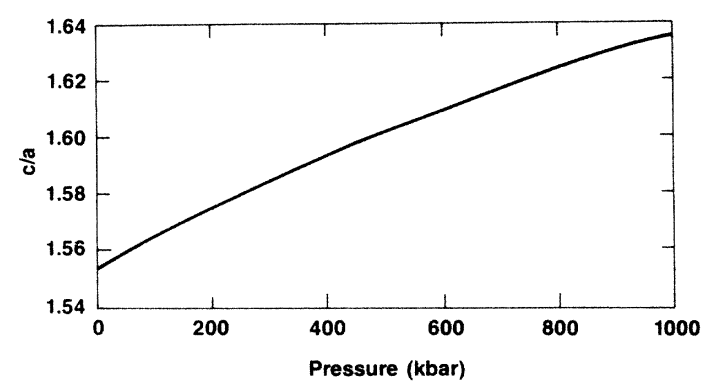

FIG. 3. The ratio of $c / a$ versus pressure as determined from Eqs. (9) and (11). The ideal value of $c / a$ is 1.6333 .

contain cubic terms in $c$ or $a$. Thus, we show only the $c / a$ ratio up to 1 Mbar. The interesting result here, but perhaps not unexpected, is that the $c / a$ value, which both experimentally and theoretical is found to be less than ideal $(c / a)_{\text {ideal }}=\sqrt{8 / 3}=1.6333 \ldots$ at zero pressure, is calculated to approach the ideal ratio at very large pressures. Intuitively one would expect that a hexagonalclose-packed crystal would have a $c / a$ ratio which deviates from the ideal value owing to anisotropic forces. As one applies a large isotropic force to the crystal, one might expect the crystal binding to be become more isotropic and for the structure to assume a more isotropic structure, i.e., an ideal hexagonal-close-packed structure. It is interesting that our calculation suggests this and, moreover, it suggests that at very high pressures the $c / a$ ratio starts to saturate near the ideal value.

\section{ELECTRONIC PROPERTIES OF RUTHENIUM}

While we have concentrated on the structural properties of ruthenium, we have also calculated the electronic properties of ruthenium in terms of its band structure. The band structure along the $c$ direction has been measured by angle-resolved photoemission ${ }^{35}$ and has been calculated previously by several workers. ${ }^{1,18-21}$ We present the band structure of ruthenium in Fig. 4 along several high sym-

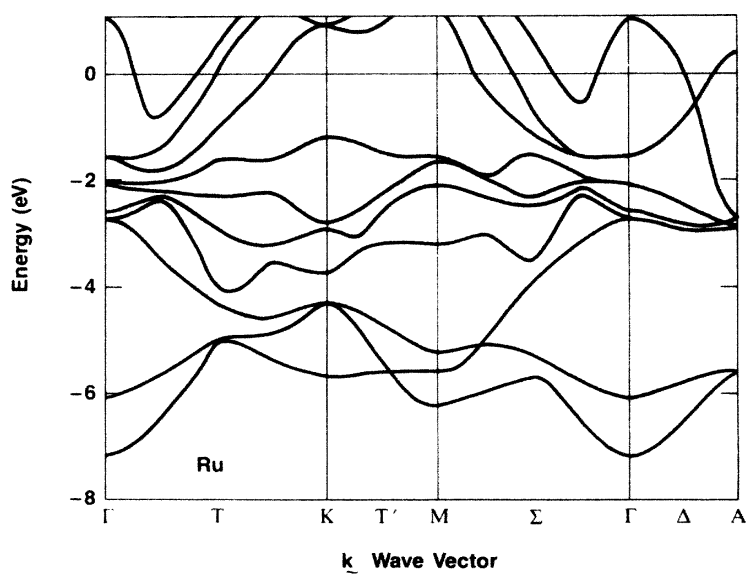

FIG. 4. Band structure of ruthenium calculated at the experimental lattice parameters. metry directions. The band structure we display is at the experimental value of the lattice constants ( $c_{\text {expt }}$ and $\left.a_{\text {expt }}\right)$. However, we have also calculated the band structure at the calculated equilibrium values and find only small differences (the eigenvalues change by less than 0.25 eV).

Perhaps the best analysis of the band structure and Fermi surface of ruthenium is from Jepsen et al. ${ }^{19}$ They used a linear muffin-tin-orbital method and a Slater-type exchange. Thus, it would not be unexpected to have some significant differences in our band structures. However, the two calculations agree quite well in terms of overall band dispersion shapes. For example, the detailed band shapes along the $T$ direction which show very dispersive and nonmonotonic band configurations are in very good agreement. In addition, although we have not done a detailed calculation of the Fermi surface, the placement of our Fermi level and energy-level crossings are nearly identical with that of Jepsen et al. ${ }^{19}$ One significant difference is the overall bandwidth. The calculation of Jepsen et al. ${ }^{19}$ yields a conduction bandwidth of approximately $8.2 \mathrm{eV}$ as contrasted with our calculated width of $7.2 \mathrm{eV}$. This is not a large discrepancy in the sense that we did not include relativistic effects which are known to increase the valence bandwidth by about $0.5 \mathrm{eV}$. Moreover, Jepsen et al. used a different exchange-correlation local-density approximation. Compared to relativistic pseudopotential calculations using the same exchange-correlation approximations, we are in better agreement. The calculation of Holzwarth and Chelikowsky ${ }^{18}$ yielded a bandwidth of approximately $7.7 \mathrm{eV}$.

With respect to experiment, our bands are in good accord along the $\Gamma-A$ direction as measured by Himpsel et al. using angle-resolved photoemission. In particular, the measured valence bandwidth is approximately $7.6 \mathrm{eV}$ and the width of the bottom two bands is about $1.5 \mathrm{eV}$ at $\Gamma$. Our calculation yields a width of $1.1 \mathrm{eV}$. The separation of the lowest two bands from the higher band complex at $A$ is about $3.0 \mathrm{eV}$ as measured and $2.6 \mathrm{eV}$ as per our calculation. At $A$ (at $-3.0 \mathrm{eV}$ relative to the Fermi level) we find a slight splitting between a fourfold degenerate level $\left(A_{3}\right)$ and a twofold degenerate level $\left(A_{1}\right)$. This splitting appears to be a function of the deviation of the $c / a$ ratio from the ideal ratio and the details of the potential. For the measured $c / a$ ratio (1.58), we find a splitting between $A_{3}-A_{1}$ of about $0.2 \mathrm{eV}$. For the calculated ratio (1.55), we find a splitting of $0.6 \mathrm{eV}$. However, the splittings are not directly attributable to the deviations of $c / a$ from its ideal value. In particular, it has been observed that this splitting is different for relativistic and nonrelativistic potentials for the same $c / a$ ratio. ${ }^{36}$

One notable discrepancy in our calculation as compared to the work of Himpsel et al. ${ }^{35}$ is their placement of a bulk band near the Fermi level. Specifically, they suggest the existence of a nearly dispersionless bulk band along the $\Delta$ direction which is within $0.3 \mathrm{eV}$ of the Fermi level. This suggestion is a variance with our present calculation, a previous relativistic pseudopotential calculation using a mixed basis ${ }^{18}$ and the work of Jepsen et al. ${ }^{19}$ At present we have no explanation for this disagreement between theory and experiment. 


\section{CONCLUSIONS}

In summary, we have examined the electronic, cohesive, and structural properties of hexagonal-close-packed ruthenium metal. We used an ab initio pseudopotential method with Gaussian orbitals to solve a single-particle Schrödinger equation and obtain the crystalline total energy. By varying the lattice parameters $(c$ and $a$ ) we were able to determine the equilibrium lattice constants, the cohesive energy, bulk modulus, derivative of the bulk modulus with pressure, and Poisson's ratio. Moreover, we were able to predict the $c / a$ ratio as a function of pressure up to $1 \mathrm{Mbar}$. We expect that the $c / a$ ratio which is less than the ideal value at zero pressure will approach and saturate at the ideal value as a function of pressure.

With respect to experiment, we find that the calculated values for the lattice parameters are in good agreement with experiment. The largest discrepancy is on the order of $2 \%$ for the $c$ value. The cohesive energy is about $15 \%$ larger than the measured value, but is in line with other local-density calculations for transition-metal cohesive energies. The bulk modulus and Poisson's ratio are both about 5\% larger than experiment.

Our band structure at the experimental lattice parameters is in accord with previous calculations for ruthenium. We find our bands are within $0.5 \mathrm{eV}$ of relativistic pseudopotential calculations and, although our bandwidths may be different, we find similar dispersions to previous muffin-tin calculations. ${ }^{19}$ With respect to angle-resolved photoemission work, ${ }^{35}$ we find ourselves in agreement with experiment with the possible exception of a measured bulk band near the Fermi level.

\section{ACKNOWLEDGMENT}

This work was supported in part by the Director, Office of Energy Research, Office of Basic Energy Sciences, Materials Sciences Division of the U.S. Department of Energy under Contract No. DE-AC03-76SF0098.
${ }^{1}$ V. L. Morruzi, J. F. Janak, and A. R. Williams, Calculated Electronic Properties of Metals (Pergamon, New York, 1978).

2J. Ihm and M. L. Cohen, Phys. Rev. B 21, 1004 (1980).

${ }^{3}$ A. K. Ray, S. B. Trickey, and A. B. Kunz, Solid State Commun. 41, 351 (1982).

${ }^{4}$ F. Averill, Phys. Rev. B 6, 3637 (1972).

${ }^{5}$ G. Bachelet, H. S. Greenside, G. A. Baraff, and M. Schluter, Phys. Rev. B 24, 4745 (1981).

${ }^{6}$ B. Harmon, W. Weber, and D. R. Hamann, Phys. Rev. B 25, 1109 (1981).

${ }^{7}$ K. M. Ho, C. L. Fu, B. Harmon, and D. R. Hamann, Phys. Rev. Lett. 49, 673 (1982).

${ }^{8}$ P. Glotzel, B. Segall, and O. K. Andersen, Solid State Commun. 36, 403 (1980).

${ }^{9}$ J. Callaway, X. Zou, and D. Bagayoko, Phys. Lett. 89A, 86 (1982); Phys. Rev. B 27, 631 (1983).

${ }^{10}$ A. Zunger and A. J. Freeman, Phys. Rev. B 15, 5049 (1977).

${ }^{11}$ J. Ihm, A. Zunger, and M. L. Cohen, J. Phys. C 12, 4409 (1979).

${ }^{12}$ M. T. Yin and M. L. Cohen, Phys. Rev. B 26, 5668 (1982).

${ }^{13}$ M. T. Yin and M. L. Cohen, Phys. Rev. Lett. 50, 2006 (1983).

14J. R. Chelikowsky and S. G. Louie, Phys. Rev. B 29, 3470 (1984).

15J. R. Chelikowsky, S. G. Louie, D. Vanderbilt, and C. T. Chan, Int. J. Quant. Chem. Symp. 18, 105 (1984).

16P. K. Lam and M. L. Cohen, Phys. Rev. B 24, 4224 (1981); M. Y. Chou, P. K. Lam, and M. L. Cohen, Solid State Commun. 42, 861 (1982); Phys. Rev. B 28, 4179 (1983), and references therein.

${ }^{17}$ C. T. Chan, D. Vanderbilt, and S. G. Louie, Phys. Rev. B 33, 2455 (1986), 34, 8791(E) (1986); C. T. Chan, D. Vanderbilt, S. G. Louie, and J. R. Chelikowsky, ibid. 33, 7941 (1986).

${ }^{18}$ N. A. W. Holzwarth and J. R. Chelikowsky, Solid State Com- mun. 53, 171 (1985).

${ }^{19}$ O. Jepsen, O. K. Andersen, and A. R. Macintosh, Phys. Rev. B 12, 3084 (1975).

${ }^{20}$ C. Q. Ma, H. Krakauer, and B. R. Cooper, J. Vac. Sci. Technol. 18, 581 (1981).

${ }^{21}$ P. J. Feibelman, Phys. Rev. B 26, 5347 (1982).

${ }^{22}$ B. A. Sexton and G. J. Somorjai, J. Catal. 46, 167 (1977).

${ }^{23}$ D. W. Goodman and J. M. White, Surf. Sci. 90, 201 (1979).

${ }^{24}$ K. Christmann, G. Ertl, and H. Shimizu, J. Catal. 61, 397 (1980).

${ }^{25}$ D. Goodman, R. D. Kelly, T. E. Madey, and J. T. Yates, Jr., J. Catal. 63, 226 (1980).

${ }^{26}$ D. R. Hamann, M. Schlüter, and C. Chiang, Phys. Rev. Lett. 43, 1494 (1979).

${ }^{27}$ D. Vanderbilt and S. G. Louie, J. Comput. Phys. 56, 259 (1984).

${ }^{28}$ L. Hedin and B. I. Lundqvist, J. Phys. C 4, 2064 (1971).

${ }^{29}$ C. L. Fu and K. M. Ho, Phys. Rev. B 28, 5480 (1983).

${ }^{30}$ O. Gunnarsson, B. I. Lundqist, and J. W. Wilkins, Phys. Rev. B 10, 1319 (1977).

${ }^{31}$ F. D. Murnaghan, Proc. Nat. Acad. Sci. U.S.A. 3, 244 (1944).

${ }^{32}$ See C. T. Chan, D. Vanderbilt, and S. G. Louie, Phys. Rev. B 34, 2455 (1986); 34, 8791(E) (1986); J. W. Davenport, R. E. Watson, and M. Weinert, ibid. 32, 4883 (1985).

${ }^{33}$ S. G. Louie, S. Froyen, and M. L. Cohen, Phys. Rev. B 26, 1738 (1982).

${ }^{34}$ Metal Handbook, 8th ed. (American Society of Metals, Cleveland, 1961), p. 10.

${ }^{35}$ F. Himpsel, K. Christmann, P. Heimann, and D. Eastman, Phys. Rev. B 23, 2548 (1981).

${ }^{36}$ N. A. W. Holwarth (private communication): For nonrelativistic potentials the splitting is approximately $0.5 \mathrm{eV}$; for relativistic potentials the splitting is less than $0.01 \mathrm{eV}$. 